\title{
Critical discussion of Tomboulis's approach to the confinement problem
}

\section{Keiichi R. Ito}

Department of Mathematics and Physics

Setsunan University

Neyagawa, Osaka 572-8508, Japan

E-mail: ito@mpg.setsunan.ac.jp

\section{Erhard Seiler*}

Max-Planck-Institut für Physik

(Werner-Heisenberg-Institut)

Föhringer Ring 6

D-80805 München, Germany

E-mail: ehs @mppmu .mpg . de

The approach to the confinement problem proposed by E.T. Tomboulis is analyzed critically. We point out some problems and discuss the chances of producing a proof by using his strategy.

8th Conference Quark Confinement and the Hadron Spectrum September 1-6 2008

Mainz, Germany

\footnotetext{
* Speaker.
} 


\section{Historical remarks}

Since this talk is about a very old issue, it seems appropriate to start with a few historical remarks to put the problem and its proposed solution by Tomboulis into context.

The confinement problem in lattice Yang-Mills theory was a hot issue in the late 1970's and early 1980's. Center vortices were identified by several authors as crucial objects $([1,2,3])$. 't Hooft proposed a confinement criterion inspired by these vortices; unlike the earlier criterion proposed by Wilson [4] and its modification by Polyakov [5] it did not involve infinitely heavy quark sources but (sourceless) central electric flux in a torus.

A little later it was proven that 't Hooft's confinement criterion implies confinement in the sense of Wilson and Polyakov [6, 7].

About the same time Tomboulis [8] came up with a charming idea how to prove that lattice Yang-Mills theory based on a nonabelian (compact, semisimple) gauge group has a nonzero string tension in 't Hooft's sense at all values of the bare coupling constant: he proposed to link by rigorous inequalities lattice Yang-Mills theory to the solution of an approximate Renormalization invented earlier by Migdal and Kadanoff (MK RG) [9, 10].

It was proven a little later that in 4 dimensions the MK RG drives lattice $S U(N)$ Yang-Mills theory, but also compact lattice QED to the strong coupling fixed point [11]. This signals confinement for these models, and is therefore misleading for the abelian model, which is known to have a deconfining transition $[12,13]$.

This fact raised problems for Tomboulis's approach, because it was not clear how his inequalities would distinguish between the groups $S U(N)$ and $U(1)$, especially since in his short letter there were no details given concerning the proof of the crucial inequalities. In fact there even remained room for doubt as to the existence of confinement in this sense in the $S U(N)$ lattice models or the analogous question of mass generation in $2 D O(N)$ spin models (see for instance [15] and references given there). In spite of these efforts as well as the efforts by others, such as K. R. Ito [16], who tried to prove mathematically the correctness of the common expectations, neither confinement for arbitrarily weak bare coupling nor its absence could be established (nor could the analogous $2 D$ problem be definitely settled). The problem remains an important open question to this day.

In 2007 Tomboulis [17] revived his old idea (with some modifications) and published a paper providing details about the purported proof. The following remarks, while critical of his work, should nevertheless not diminish his credit for having revived interest in this old, important but neglected and unsolved problem. A more detailed discussion can be found in our paper [18].

\section{Sketch of Tomboulis's strategy}

The goal of Tomboulis's strategy, for simplicity for the gauge group $S U(2)$, is to establish the spreding of central magnetic flux on a torus $\Lambda$ of dimensions $L_{1} \times L_{2} \times L_{3} \times L_{4}$ :

$$
\frac{Z_{\Lambda}^{(-1)}}{Z_{\Lambda}} \geq \exp \left[-c L_{2} L_{3} e^{-\alpha L_{1} L_{4}}\right] \quad \text { for } \quad L_{1} L_{4} \gg \log \left(L_{2} L_{3}\right)
$$


where $Z_{\Lambda}^{(-1)}$ has twisted boundary conditions in the (12) direction. (2.1)is supposed to follow from

$$
\frac{Z_{\Lambda}^{(-)}}{Z_{\Lambda}} \geq \frac{Z_{M K T}^{(-)}(n)}{Z_{M K T}(n)} .
$$

Here $Z_{M K T}(n), Z_{M K T}^{(-)}(n)$ are the partition functions under the $n$-fold iteration of the 'MKT' decimation which is Tomboulis's modification of the MK RG.

If we assume for a moment that inequality (2.2) holds and the MKT iteration leads eventually into the strong coupling regime, inequality (2.1) follows, and this implies electric flux string formation and confinement in the sense that 't Hooft's string tension $\sigma_{t H}$ satisfies

$$
\sigma_{t H}>0 \quad \forall g^{2},
$$

where $g$ denotes the bare coupling constant.

One question that arises immediately is whether Ito's result, establishing flow to the strong coupling fixed point, also holds for Tomboulis's modification, which depends on an additional parameter $r=1-\varepsilon, \varepsilon>0$. We found that $r<1$ has the same effect as increasing the dimension and therefore for weak coupling the flow actually goes towards the weak coupling fixed point.

\section{The fundamental issue}

As remarked before, the MK RG in $4 D$ shows no structural difference between abelian (such as $U(1)$ ) and nonabelian (such as $S U(N)$ ) models: the flow is always attracted by the strong coupling fixed point. This was already pointed out in the seminal paper [10], where this insight was actually traced to Wilson's 1976 Cargèse lectures; as remarked, a proof of this fact was given by Ito [11].

This means that the original comparison argument given by Tomboulis has to fail for $U(1)$, because the $4 D U(1)$ model has vanishing string tension for sufficiently weak coupling. In fact, any similar argument that does not explicitly make use of the nonabelian nature of the gauge group has to fail.

\section{Technical points}

(a) The parameter $r$.

The MKT decimation proceeds as follows: one starts with the character expansion of the plaquette coupling function (Gibbs factor)

$$
f(U) \equiv \exp A_{p}(U)=F_{0}\left[1+\sum_{j \neq 0}(2 j+1) c_{j}(\beta) \chi_{j}(U)\right] .
$$

In essence the decimation amounts to alternating raising the coupling function to the power $2^{D-2}$ and raising the Fourier coefficients $c_{j}$ to the power $4 r$. Explicitly

$$
f^{(n)}(U) \mapsto \frac{f^{(n)}(U)^{4}}{\int f^{(n)}(U)^{4} d U} \equiv g^{(n)}(U)
$$




$$
\begin{gathered}
g^{(n)}(U)=1+\sum_{j>0}(2 j+1) c_{j}(n) \chi_{j}(U) \\
f^{(n+1)}(U)=1+\sum_{j>0}(2 j+1) c_{j}(n)^{4 r} \chi_{j}(U) .
\end{gathered}
$$

Equality of the two exponents $2^{D-2}$ and $4 r$ would mean that one is working in the critical dimension $D_{c}$ and one finds easily

$$
D_{c}=4+\frac{\ln r}{\ln 2}<4,
$$

so that with $r<1$ in $4 D$ one is above the critical dimension and has to expect a phase transition. This is indeed the case; we have run the iteration for $r=0.9$ and two close values of $\beta \equiv 2 / g^{2}$ and found a bifurcation of the flow: for $\beta=4.79$ the flow is attracted to the weak coupling fixed point, whereas for $\beta=4.80$ is flows to the strong coupling fixed point. The fact that for weak coupling the flow converges to the weak coupling fixed point can also be seen in a simple Gaussian approximation.
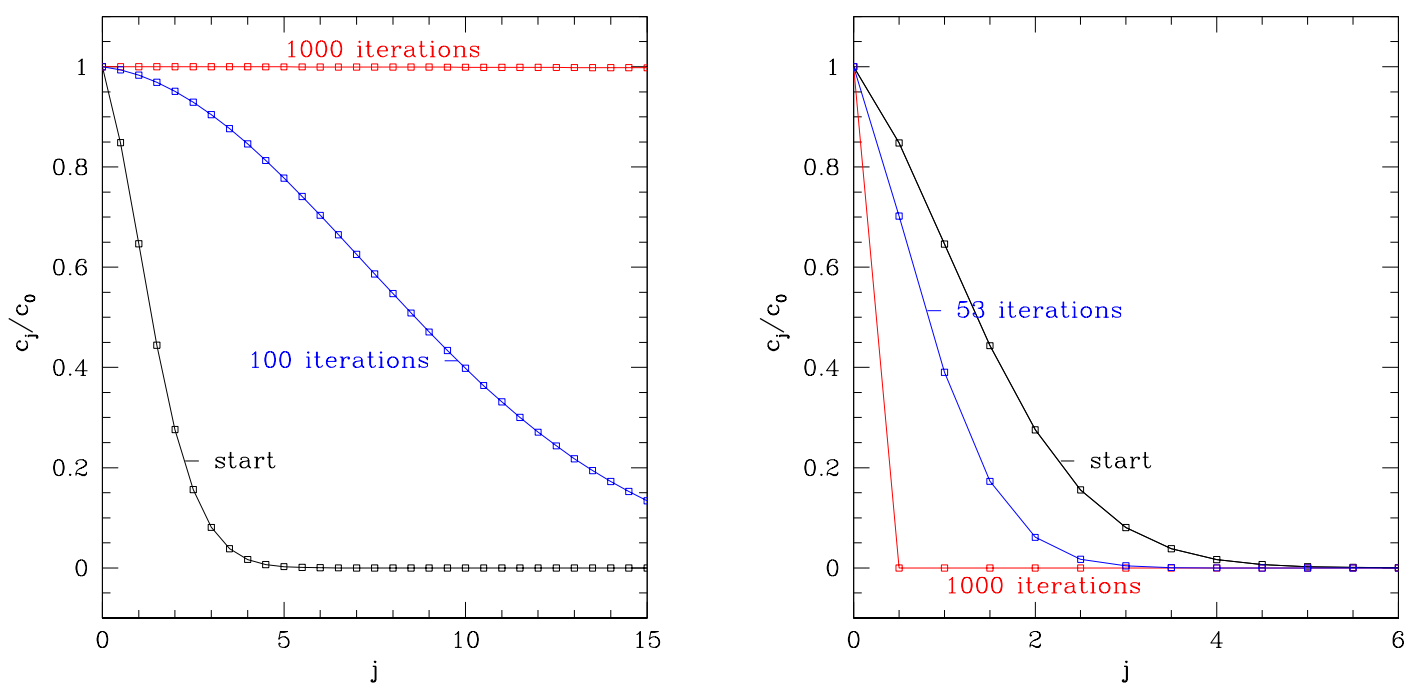

Figure 1: Evolution of $c_{j} / c_{0}$ under Tomboulis' modified MK RG with $r=0.9 . \beta=4.80$ (left plot), $\beta=4.79$ (right plot); lines drawn to guide the eye.

(b) Existence of a common interpolation parameter $\alpha^{*}$ for $Z$ and $Z^{(-)}$.

This is an essential point in Tomboulis's strategy. He has to find for all $n$ an $\alpha(n)^{*} \leq 1-\delta$ such that

$$
\frac{Z_{\Lambda}^{(-)}}{Z_{\Lambda}}=\frac{Z_{\Lambda}^{(-)}\left(\left\{\alpha^{*}(n) c_{j}(n)\right\}\right.}{Z_{\Lambda}\left(\left\{\alpha^{*}(n) c_{j}(n)\right\}\right)}
$$

His argument (in Appendix $C$ of [17]), based on the implicit function theorem, is flawed. He introduces a certain function $\Psi(\lambda, t)$ in terms of interpolated partition functions and is looking for a $t(\lambda)$ such that

$$
\Psi(\lambda, t)=0 .
$$

There is a solution $t_{0}$ at $\lambda=0$, but the sought after $\alpha^{*}$ would emerge from $t(1)$. Tomboulis is able to show that $\frac{\partial}{\partial t} \Psi(\lambda, t) \neq 0$, so by the implicit function theorem there is a solution near $\lambda=0$. But the 
information is not sufficient to allow the extension to $\lambda=1$, as shown by a simple counterexample due to T. Kanazawa [14]:

$$
\Psi(\lambda, t) \equiv e^{-t}-1+2 \lambda
$$

which has the solution $t(\lambda)=-\log (1-2 \lambda)$.

\section{Can the problems be fixed?}

The choice of the parameter $r$ is very subtle, because one has to make sure of two things: (1) the decimation has to run into the strong coupling fixed point and (2) $r$ has to be kept away from 1 , as is stressed in [17]. This second issue is not discussed in [17] in a quantitative way, while the issue (1) is not addressed at all. Tomboulis hinted orally at the option of making $r$ dependent on $n$, the number of the iterations, but exactly how this would have to be done remains unclear.

In this respect the case of $U(1)$ is instructive: for $r=1$ the common interpolation parameter $\alpha^{*}$ cannot exist, because it would imply the existence of a nonvanishing string tension at all values of the bare coupling, in contradiction with proven facts $([12,13])$.

Quite generally, we think that any strategy based on a Migdal-Kadanoff type decimation is very unlikely to succeed, because these hierarchical approximations do not show any structural difference between abelian (like $U(1)$ ) and nonabelian (like $S U(2))$ models.

\section{References}

[1] T. Yoneya, Nucl. Phys. B144 (1978) 195.

[2] G. 't Hooft, Nucl. Phys. B138 (1978) 1; B153 (1979) 141.

[3] G. Mack and V. B. Petkova, Ann. of Physics 123 (1980) 117.

[4] K. Wilson, Phys. Rev. D10 (1975) 2445.

[5] Phys.Lett.B 72 (1978) 477.

[6] C. Borgs and E. Seiler, Commun.Math.Phys. 91 (1983) 329.

[7] E. T. Tomboulis amd L.G. Yaffe, Commun.Math.Phys. 100 (1985,313.

[8] E. T. Tomboulis, Phys.Rev.Lett. 50 (1983) 885.

[9] A. A. Migdal, JETP 69 (1975) 810; L. Kadanoff, Ann. Phys. 100 (1976) 359.

[10] J. V. Jose, L. P. Kadanoff, S. Kirkpatrick, D. E. Nelson, Phys.Rev. B16 (1977) 1217.

[11] K. R. Ito, Phys.Rev.Lett.54 (1985) 2383; Phys.Rev.Lett.55 (1985) 558;

[12] A. Guth, Phys. Rev. D21, (1980) 2291.

[13] J. Fröhlich and T. Spencer, Commun.Math.Phys. 83 (1982) 411.

[14] T. Kanazawa, private communication to K .R. Ito.

[15] E.Seiler, RIMS Kokyuroku (Res.Inst.Math.Sci., Kyoto University) 1386 (2004), 193; arXiv hep-th/0312015.

[16] K. R. Ito, Lett.Math.Phys.37 (1996) 349.

[17] E. T. Tomboulis, Confinement for all values of the coupling in 4D SU(2) gauge theory, arXiv:0707.2179[hep-th]; these proceedings.

[18] K. R. Ito and E. Seiler, arXiv:0803.3019 [hep-th]. 